\title{
Elevated level of interleukin-29: A proinflammatory role in synovial inflammation of rheumatoid arthritis
}

\author{
Dalia A. Shaheen ${ }^{1}$, Hisham M. Habib ${ }^{2}{ }^{*}$ \\ ${ }^{1}$ Department of Medical Biochemistry, Faculty of Medicine, Mansoura University, Egypt \\ ${ }^{2}$ Department of Rheumatology and Rehabilitation, Faculty of Medicine, Mansoura University, Egypt
}

Email address:

hesham_habib@yahoo.com (H. M. Habib)

\section{To cite this article:}

Dalia A. Shaheen, Hisham M. Habib. Elevated Level of Interleukin-29: A Proinflammatory Role in Synovial Inflammation of Rheumatoid Arthritis. Advances in Biochemistry. Vol. 2, No. 2, 2014, pp. 29-33. doi: 10.11648/j.ab.20140202.11

\begin{abstract}
Background: The multiple pro-inflammatory cascades described in RA lead to persistent synovitis, resulting in articular cartilage and bone damages. The up-regulation of interferon-inducible genes has been found in the synovial lining regions and may play an important role in the pathogenesis of RA. Objective: The aim of the current study is to examine the expression of IL-29 and its receptors IL-28R $\alpha$ in blood and synovial fluid (SF) in RA patients and investigate the correlation of IL-29 with disease activity and other laboratory characters of RA. Methods: The study was carried out on 108 RA patients, 70 OA patients and 80 healthy control subjects. Clinical assessment of disease activity in RA patients and disease activity score with DAS28 had been done. IL29 was detected in the serum and synovial fluid of all samples by ELISA. Real time PCR was used for human IL-29 and IL-28R $\alpha$ expression from PBMC. Results: expression of IL-29 mRNA was significantly higher in RA PBMCs when compared to OA and HC $(132 \pm 13.8,49 \pm 11.2,33.3 \pm 10.5$; respectively P $<0.05)$. Similarly, IL-28R $\alpha$ mRNA expression was significantly higher in RA PBMCs when compared to OA and HC (30.5 $\pm 9.7,13.3 \pm 4.5,8.5 \pm 3.3$; respectively P $<0.05)$. The mean level of IL-29 in SF was increased in RA and found also much higher in RA $(17.29 \pm 10.19 \mathrm{pg} / \mathrm{ml})$ than $\mathrm{OA}(8.33 \pm 3.89 \mathrm{pg} / \mathrm{ml})(\mathrm{P}=0.01)$. There is a significant correlation between serum levels of circulating IL- 29 and DAS28, CRP, ESR, RF, and anti-CCP. Conclusion: our data have presented new evidence that IL-29 may contribute to RA pathogenesis.
\end{abstract}

Keywords: IL29, Rheumatoid Arthritis, Synovial Inflammation

\section{Introduction}

Rheumatoid arthritis (RA), the most common form of chronic inflammatory arthritis, is characterized by persistent synovial inflammation, systemic inflammation, and autoantibodies. The multiple proinflammatory cascades described in RA lead to persistent synovitis, resulting in articular cartilage and bone damages [1]. The proinflammatory cytokines tumor necrosis factor (TNF)alpha, interleukin (IL)-1-beta, and IL-6, produced by synovial cells and infiltrating cells, actively contribute to RA Pathogenesis [1,2]. Recently, the upregulation of interferon-inducible genes has been found in the synovial lining regions and whole blood of patients with RA, suggesting that interferons (IFNs) may also play an important role in the pathogenesis of RA [3, 4].

The classical IFN family cytokines, such as types I and II IFNs, are known to play an important role in both innate and adaptive immune responses during viral infection and autoimmune inflammation. Type III IFNs, or IFN- $\lambda$ s are a newly described member of the IFN family, including IFN- $\lambda 1$ (IL-29), IFN- $\lambda 2$ (IL-28A) and IFN- $\lambda 3$ (IL-28B) [5,6]. IL-28A and IL-28B proteins are $95 \%$ identical while IL-29 shares only $80 \%$ amino acid identity with IL- 28A or IL-28B [7]. Each member of type III IFNs signals through the heterodimeric receptor complex, including the IFN- $\lambda \mathrm{R}$ (IL-28R $\alpha$ ) chain and IL-10 receptor type 2 (IL-10R2) chain $[5,6,8]$. While most cells express the IL-10R2 subunit, the expression of IL-28R $\alpha$ appears to be much more cell specific [6].

Type III IFNs signal through its receptors inducing antiviral, antiproliferative, antitumor and immune responses [9-11]. Although the antiviral effects of IL-28A and IL-29 have been studied extensively during the past decade, the immunoregulatory role of type III IFNs in immune cells is still poorly understood.

The aim of the current study is to detect the serum and synovial fluid level of IL-29 in RA patients. Furthermore, we aimed to examine the expression of IL-29 and its receptors IL- 
$28 \mathrm{R} \alpha$ in blood of RA patients. Finally, we investigate the effect of IL-29 on RA disease activity.

\section{Material and Methods}

\subsection{Patients}

In our study, we recruited 108 consecutive patients diagnosed as rheumatoid arthritis according to American College of Rheumatology (ACR)/European League Against Rheumatism (EULAR) 2009 diagnostic criteria [12]. All rheumatoid arthritis (RA) patients were recruited from the "baseline" and had not yet been treated with diseasemodifying anti-rheumatic drugs (DMARDs) and/or steroids before their blood samples were collected. They were collected over a period from July 2011 to August 2013. Seventy osteoarthritis (OA) patients and eighty healthy control subjects (HC) were recruited randomly and voluntarily served as disease and healthy controls respectively. The protocol for the research project has been approved by a suitably constituted Ethics Committee of the hospital and that it conforms to the provisions of the World Medical Association's Declaration of Helsinki.

An informed consent has been obtained for all investigations on subjects of the study (and patient anonymity has been preserved).

\subsubsection{Clinical Assessment}

For clinical assessment of disease activity in all RA patients, a disease activity score with reduced joint count (DAS28) has been used. A cut-off point of DAS28 $\leq 3.2$ has been proposed that patient is in clinical remission with values $>5.1$ was representing high disease activity [11].

\subsection{Laboratory Investigations}

Blood samples were collected from all RA, OA patients and HCs. SF samples were obtained from 48 RA and 33 OA patients.

Ten milliliters of venous blood was collected from each subject and divided into three samples. The first one was used for blood chemistry analysis erythrocyte sedimentation rate (ESR), C-reactive protein (CRP), anticyclic citrullinated peptides (CCP) and rheumatoid factor (RF). They are analyzed immediately. The second blood sample was used for immunoassay of serum human IL 29 levels and the sera were stored at $<-70{ }^{\circ} \mathrm{C}$ until thawed for the assays. The third one is used for expression of Human IL 29 and Human IL-28R $\alpha$ from PMNC.

5-10 milliliters of SF was collected from each subject and stored at $<-70{ }^{\circ} \mathrm{C}$ until thawed for the immunoassays.

\subsubsection{ELISA}

IL29 was detected by LEGEND MAX ${ }^{\text {TM }}$ Human IL-29 ELISA Kit (San Diego, CA 92121). Read absorbance at $450 \mathrm{~nm}$ within 30 minutes. The level of IL29 (expressed as $\mathrm{pg} / \mathrm{ml}$ ) will be correlated with the laboratory values, of ESR, CRP, anti-CCP and RF in serum of RA patients.

\subsubsection{Real-time PCR}

Total RNA was extracted from PBMC using TRIzol (Invitrogen, Carlsbad, CA, USA). (QIAamp RNA Blood Mini Kit, QIAGEN, Hilden, Germany; Cat. No. 52304) according to the manufacturer instructions.

Thereafter, RNA was reversed transcribed from $2.5 \mu \mathrm{g}$ total RNA according to the manufacturer's instructions, using random hexamers (Invitrogen). cDNA was used for real-time PCR with the help of StepOne ${ }^{\mathrm{TM}}$ real-time PCR system (Applied Biosystems, Foster City, CA, USA).

The sequences of oligonucleotide primers used in the PCR for detection of IL29 and IL-28Ra expression levels, were as follows:

- For Human IL-29: Forward is 5'GCCATGGCTGCAGCTTGGAC-3' and Reverse is 5'GGTGGACTCAGGGTGGGTTGAC-3'

- For of Human IL-28R $\alpha$ : Forward is 5'CCTCCCCAGAATGTGACGC3' and Reverse is 5' GGAGCCATGTCAGGTACACG 3'

- For Human GAPDH sense: Forward 5'GAAGATGGTGATGGGATTTC-3' and Reverse 5'GAAGGTGAAGGTCGGAGT-3'.

The amplification scheme used was as follows: $94^{\circ} \mathrm{C}$ for 5 minutes, then 35 cycles of $94^{\circ} \mathrm{C}$ for 45 seconds, $60^{\circ} \mathrm{C}$ for 45 seconds, and $72^{\circ} \mathrm{C}$ for 45 seconds, followed by $72^{\circ} \mathrm{C}$ for 7 minutes and then $4^{\circ} \mathrm{C}$. Data from the reaction were collected and analyzed. The relative copy numbers of gene expression were quantitated using the comparative threshold cycle (ct ) method.

\subsection{Statistical Analysis}

Statistical analyses were performed with SPSS software (SPSS, Chicago IL, USA). Data were expressed as mean \pm SD. Differences between two groups were detected with Student's t test for parametric data and Mann-Whitney U test for nonparametric data. The Pearson correlation test was used to evaluate the correlation between serum IL-29 levels and laboratory values and clinical features. $\mathrm{P}$ values $<0.05$ was considered as significant.

\section{Results}

The Demographic, Clinical and laboratory characteristics of both patients and controls subjects are summarized in Table 1. In general, the study group comprised 108 RA patients (80 females, 28 males) with a mean age of 37.5 (range 19-62) years, 70 OA patients (53 females, 17 males) with a mean age of 64.5(range 55-77) and $80 \mathrm{HC}$ subjects (58 females, 22 males) with a mean age of 44.5 (range: 23 63). There was no statistically significant difference in the age and gender of the studied groups. DAS28 in RA patients was found to be $4.1 \pm 1.38$.

\subsection{Unregulated Expression of IL-29 and its Receptor Transcripts in PBMCs from RA Patients}

To explore whether IL-29 was involved in the 
pathogenesis of RA, we examined the expression of the IL29 mRNA. Since, the biological functions of IL-29 depend mainly on its receptor; we also examined the expression of its receptors IL-28R $\alpha$ mRNA in PBMC by real-time PCR.

Results showed that expression of IL-29 mRNA was significantly higher in RA PBMCs when compared to OA and $\mathrm{HC}(132 \pm 13.8,49 \pm 11.2,33.3 \pm 10.5$; respectively $\mathrm{P}$ $<0.05)$. Similarly, IL-28R $\alpha$ mRNA expression was significantly higher in RA PBMCs when compared to OA and $\mathrm{HC}(30.5 \pm 9.7,13.3 \pm 4.5,8.5 \pm 3.3$; respectively $\mathrm{P}<0.05)$. (Tables (2) A and (2) B).

Table 1. Demographic, Clinical and laboratory characteristics in patients with rheumatoid arthritis (RA), osteoarthritis (OA) and healthy control (HC) subjects.

\begin{tabular}{llll}
\hline Variable & RA $(\mathbf{n}=\mathbf{1 0 8})$ & OA $(\mathbf{n}=\mathbf{7 0})$ & HC $(\mathbf{n}=\mathbf{8 0})$ \\
\hline Age $(\mathrm{y})$ & $37.5 \pm 12.6$ & $64.5 \pm 17.3$ & $44.5 \pm 16.6$ \\
Sex (female/male) & $80 / 28$ & $53 / 17$ & $58 / 22$ \\
Age at onset $(\mathrm{y})$ & $34.5 \pm 11.5$ & - & - \\
Disease duration & $26 \pm 9$ & - & - \\
(m) & & & - \\
Number of tender & $7.50 \pm 2$ & - & - \\
joints & & & - \\
Number of swollen & $3 \pm 1$ & - & - \\
joints & $44 \pm 11.6$ & - & - \\
ESR (mm/h) & $11.55 \pm 3.3$ & - & \\
CRP (mg/l) & $4.1 \pm 1.38$ & & $25.82 \pm 5.79$ \\
DAS28 & $56.8 \pm 17.6$ & - & \\
RF (U/I) & $83 / 25$ & & \\
CCP (+ve/-ve) & $26.51 \pm 3.33$ & $29.07 \pm 4.73$ & \\
BMI (kg/m2) & & & \\
\hline
\end{tabular}

BMI, body mass index; CCP, anti-cyclic citrullinated peptides CRP; Creactive protein; DAS28, 28-joint Disease Activity Score; ESR, erythrocyte sedimentation rate; RF, rheumatoid factor.

Table (2).A. Serum IL-29 mRNA relative expression in different studied groups.

\begin{tabular}{lll}
\hline Group & Serum IL-29 levels $(\mathbf{p g} / \mathbf{m l})$ Mean \pm SD & P \\
\hline RA $(\mathrm{N}=108)$ & $132 \pm 13.8$ & \\
OA $(\mathrm{N}=70)$ & $49 \pm 11.2$ & $\mathrm{P}<0.05$ \\
$\mathrm{HC}(\mathrm{N}=80)$ & $33.3 \pm 10.5$ & \\
\hline
\end{tabular}

RA, rheumatoid arthritis; OA, osteoarthritis; $\mathrm{HC}$, healthy control.

Table (2).B. IL-28R a mRNA relative expression in different studied groups.

\begin{tabular}{lll}
\hline Group & IL-28R $\boldsymbol{\alpha}$ Mean \pm SD & P \\
\hline $\mathrm{RA}(\mathrm{N}=108)$ & $30.5 \pm 9.7$ & \\
$\mathrm{OA}(\mathrm{N}=70)$ & $13.3 \pm 4.5$ & $\mathrm{P}<0.05$ \\
$\mathrm{HC}(\mathrm{N}=80)$ & $8.5 \pm 3.3$ & \\
\hline
\end{tabular}

RA, rheumatoid arthritis; OA, osteoarthritis; HC, healthy control.

\subsection{Increased Serum and SF Levels of IL-29 in RA Patients}

To disclose the protein level of IL-29 in serum and SF, we measured the concentrations of IL-29 in the serum of RA patients and $\mathrm{HC}$ and in SF from patients with RA and OA. Our results showed that serum levels of circulating IL-29 were significantly higher in RA $(21.66 \pm 12.19 \mathrm{pg} / \mathrm{ml})$ than those in OA patients $(6.33 \pm 3.41 \mathrm{pg} / \mathrm{ml}, \mathrm{P}<0.005) \mathrm{HC}(4.32$ $\pm 2.49 \mathrm{pg} / \mathrm{ml}, \mathrm{P}<0.0005)$. The mean value of IL-29 level in $\mathrm{SF}$ was increased in RA and found also much higher in RA $(17.29 \pm 10.19 \mathrm{pg} / \mathrm{ml})$ than $\mathrm{OA}(8.33 \pm 3.89 \mathrm{pg} / \mathrm{ml})(\mathrm{P}=0.01)$. (Tables (3) A and (3) B).

Table (3).A. IL-29 level in serum in the studied groups.

\begin{tabular}{lll}
\hline Group & Serum IL-29 $(\mathbf{p g} / \mathbf{m l})$ Mean \pm SD & P \\
\hline $\mathrm{RA}(\mathrm{N}=108)$ & $21.66 \pm 12.19$ & \\
$\mathrm{OA}(\mathrm{N}=70)$ & $6.33 \pm 3.41$ & $\mathrm{P}<0.005$ \\
$\mathrm{HC}(\mathrm{N}=80)$ & $4.32 \pm 2.49$ & \\
\hline
\end{tabular}

RA, rheumatoid arthritis; OA, osteoarthritis; HC, healthy control

Table (3).B. IL-29 level in synovial fluid in RA and OA patients.

\begin{tabular}{lll}
\hline Group & Serum IL-29(pg/ml) Mean \pm SD & P \\
\hline $\mathrm{RA}(\mathrm{N}=48)$ & $17.29 \pm 10.19$ & $\mathrm{P}<0.01$ \\
$\mathrm{OA}(\mathrm{N}=33)$ & $8.33 \pm 3.89$ & \\
\hline
\end{tabular}

RA, rheumatoid arthritis; OA, osteoarthritis

\subsection{Relationship between Circulating IL-29 Levels and Disease Activity in RA Patients}

We investigated the correlations between IL-29 and 28joint Disease Activity Score (DAS28) as well as laboratory characteristics of RA patients, including CRP, ESR, RF, and anti-CCP. Our results revealed that there is a significant positive correlation between serum level of circulating IL29 and DAS28, CRP, ESR, RF, and anti-CCP (Table4). When RA patients were divided into active and inactive groups according to DAS28 score, it was found that active group revealed moderately statistically significant higher serum IL-29 mRNA and protein levels than inactive group (Table5).

Table (4). Correlation between IL-29 versus RA disease activity and laboratory characteristics.

\begin{tabular}{ccc}
\hline Variables & \multicolumn{3}{c}{ IL-29 } \\
& r & P \\
\hline DAS28 & 0.41 & $<0.01$ \\
CRP & 0.33 & $<0.005$ \\
ESR & 0.55 & $<0.001$ \\
RF & 0.39 & $<0.05$ \\
Anti-CCP & 0.43 & $<0.001$ \\
\hline
\end{tabular}

DAS28, disease activity score 28; CRP, c-reactive protein; ESR, erthocytic sedimentation rate; RF, rheumatoid factor; Anti-CCP, anti-cyclic citrullinated peptides.

Table (5). IL-29 ( $\mathrm{pg} / \mathrm{ml})$ in active versus inactive RA patients.

\begin{tabular}{lll}
\hline Active RA group & Inactive RA group & P \\
\hline $24.56 \pm 16.22$ & $14.35 \pm 10.14$ & $<0.01$ \\
\hline
\end{tabular}

\section{Discussion}

Pro-inflammatory cytokines promote inflammation, osteoclast formation and activity in bone-degenerative diseases such as rheumatoid arthritis and periodontitis. IL29 is a new member of type III IFN family of cytokines that has been to have antiviral activity, antigrowth activity 
including proapoptotic and antiproliferative activities [6,11.13]. However its immunoregulatory role in immune cells is still poorly understood. In the present study we demonstrate the role of IL-29 in the pathogenesis of RA.

The present report shows that the newly discovered cytokine IL-29 is expressed in the blood of RA patients as the mRNA levels of IL-29 and its specific receptor IL-28R $\alpha$ in PBMC were significantly higher in RA than those in HC. Levels of IL-29 were significantly elevated in RA serum compared to those of OA and HC. Also, IL-29 levels were significantly higher in RA SF than those in OA SF. Moreover, our results show a significant difference of IL29 level in blood between RA patients with active and inactive disease. Also serum IL-29 protein levels were positively correlated with DAS28, RF levels, CRP, ESR, and anti-CCP. These data suggest that IL-29 expression is dysregulated in RA patients, and may contribute to its pathogenesis aside the disease activity.

Our results were in agreement with a recent study of Wang et al [14], who demonstrated that mRNA levels of IL-29 and its specific receptor IL-28R $\alpha$ in PBMC were significantly higher in RA than those in HC. Also they reported that the levels of IL-29 were greatly elevated in RA serum compared with $\mathrm{HC}$ and significantly higher in RA SF than that in OA SF. However they showed insignificant difference of IL-29 level in blood between RA patients with active and inactive disease.

Taken together, our data provide valid evidence that IL29 could play a role in the pathogenesis of RA. The pathobiology of RA is multifaceted and involves T cells, B cells and the complex interaction of many proinflammatory cytokines, including TNF- $\alpha$ and IL-6. These cytokines are messengers that activate and differentiate effector cells that cause local and systemic symptoms associated with this disease [15-17]. Through complex signal pathways, these cytokines activate genes associated with inflammatory responses, including additional cytokines and matrix metalloproteinases (MMPs) involved in tissue degradation [18].

IL-29 has been shown to activate monocytes and macrophages to produce a restricted panel of cytokines [19, 20]. IL-29 is able to enhance phylogenetically conserved molecules that recognize pathogen-associated molecular patterns and eliminate invading pathogens by activation of immune responses known as, Toll-like receptors (TLR2, TLR3 and TLR4) expression in RA synovial fibroblasts ( RA-FLS), which by turn leads to increased production of IL-6 and IL-8[19], suggesting autoamplification of inflammation in RA-FLS through interaction of TLRs and cytokines. Also Wang et al reported that recombinant human IL-29 (rIL-29) could activate human synovial fibroblasts to produce cytokines IL-6, IL-8 and MMP-3[14].

On the other hand, Jordan et al reported that IL-29 is able to induce high levels of IL-6 and IL-8, in the absence of TLR ligands, by human monocytes [20]. Also Xu et al, reported that fibroblast-like synoviocytes of RA patients (RA-FLSs) are responsive to IL-29 and may release IL-6 and IL-8 in the absence of TLR ligands [19]. In addition, they demonstrated that the protein expression of IL- 6 and IL-8 in RA-FLSs were increased after IL-29 stimulation [19].

IL-6 is a multitarget cytokine with activity relevant to RA. At the affected joints, IL-6 has a pivotal role in the inflammatory process, in osteoclast-mediated bone resorption, and in synovitis [21]. IL-6, in particular, acts directly on neutrophils through membrane-bound IL-6R, which in turn contributes to inflammation and joint destruction by secreting proteolytic enzymes and reactive oxygen intermediates [22]. Moreover IL-6 induces acutephase proteins and contributes to the systemic manifestations of RA. In addition, IL- 6 is contributed to the induction and maintenance of the autoimmunity through B-cell activation and Th17 cell differentiation [21]. Also cartilage degradation in RA occurs when TNF- $\alpha$, IL-1 and IL-6 activate synoviocytes, resulting in the secretion of MMPs into the SF $[18,23]$.

\section{Conclusion}

In summary, our data have presented new evidence that IL-29 may contribute to RA pathogenesis. Further studies addressing the IL-29 in synovial tissue and its underlying molecular mechanisms will provide a fuller understanding of a pathological role of IL-29 in RA development as well as disease activity.

\section{References}

[1] Scott DL, Wolfe F, Huizinga TW. Rheumatoid arthritis. Lancet 2010, 376: 1094-1108.

[2] Brennan FM, McInnes IB. Evidence that cytokines play a role in rheumatoid arthritis. J Clin Invest 2008, 118: 35373545 .

[3] Yoshida S, Arakawa F, Higuchi F, Ishibashi Y, Goto M, et al. Gene expression analysis of rheumatoid arthritis synovial lining regions by cDNA microarray combined with laser microdissection: up-regulation of inflammation-associated STAT1, IRF1, CXCL9, CXCL10, and CCL5. Scand J Rheumatol 2012, 41:170-179.

[4] Higgs BW, Zhu W, Richman L, Fiorentino DF, Greenberg SA et al. Identification of activated cytokine pathways in the blood of systemic lupus erythematosus, myositis, rheumatoid arthritis, and scleroderma patients. Int J Rheum Dis 2012, 15:25-35.

[5] Witte K, Witte E, Sabat R, Wolk K: IL-28A, IL-28B, and IL-29: promising cytokines with type I interferon-like properties. Cytokine Growth Factor Rev 2010, 21:237-251.

[6] Kotenko SV: IFN- $\lambda$ s. Curr Opin Immunol 2011, 23:583-590.

[7] Diegelmann J, Beigel F, Zitzmann K, Kaul A, Göke B,et al. Comparative analysis of the lambda-interferons IL-28A and IL-29 regarding their transcriptome and their antiviral properties against hepatitis C virus. PLoS One. 2010, Volume 5 | Issue 12 | e15200 
[8] Honda K, Takaoka A, Taniguchi T. Type I interferon (corrected) gene induction by the interferon regulatory factor family of transcription factors. Immunity. 2006;25:349-360

[9] Donnelly RP, Kotenko SV: Interferon- $\lambda$ : a new addition to an old family. J Interferon Cytokine Res 2010, 30:555-564.

[10] Zhang L, Jilg N, Shao RX, Lin W, Fusco DN, et al: IL28B inhibits hepatitis $\mathrm{C}$ virus replication through the JAK-STAT pathway. J Hepatol 2011, 55:289-298.

[11] Liu MQ, Zhou DJ, Wang X, Zhou W, Ye L, et al: IFN- $\lambda 3$ inhibits HIV infection of macrophages through the JAKSTAT pathway. PLoS One 2012, 7:e35902.

[12] van Tuyl LH, Vlad SC, Felson DT, Wells G, Boers M: Defining remission in rheumatoid arthritis: results of an initial American College of Rheumatology/European League Against Rheumatism consensus conference. Arthritis Rheum 2009, 61:704-710.

[13] Witte K, Witte E, Sabat R, Wolk K. IL-28A, IL-28B, and IL29: promising cytokines with type I interferon-like properties. Cytokine Growth Factor Rev. 2010 Aug;21(4):237-51. doi: 10.1016/j.cytogfr.2010.04.002. Epub 2010 Jul 23.

[14] Wang F, Xu L, Feng X, Guo D, Tan W, et al. Interleukin-29 modulates proinflammatory cytokine production in synovial inflammation of rheumatoid arthritis. Arthritis Res Ther. 2012 Oct 19; 14(5):R228.

[15] McInnes IB, Schett G. Cytokines in the pathogenesis of rheumatoid arthritis. Nat Rev Immunol 2007; 7:429- 42.

[16] Firestein GS. Evolving concepts of rheumatoid arthritis. Nature 2003; 423:356- 61.

[17] Choy E. Understanding the dynamics: pathways involved in the pathogenesis of rheumatoid arthritis. Rheumatology 2012;51:v3- v11 doi:10.1093/rheumatology/kes113

[18] Smolen JS, Steiner G. Therapeutic strategies for rheumatoid arthritis. Nat Rev Drug Discov 2003;2:473- 88.

[19] Xu L, Feng X, Tan W, Gu W, Guo D, et al. IL-29 enhances Toll-like receptor-mediated IL-6 and IL- 8 production by the synovial fibroblasts from rheumatoid arthritis patients. Arthritis Res Ther. 2013 Oct 29; 15(5):R170. doi: 10.1186/ar4357.

[20] Jordan WJ, Eskdale J, Boniotto M, Rodia M, Kellner D, et al: Modulation of the human cytokine response by interferon $\lambda-1$ (IFN- $\lambda 1 /$ IL-29). Genes Immun 2007, 8:13-20.

[21] Hashizume.M and Mihara.M: The Roles of Interleukin-6 in the Pathogenesis of Rheumatoid Arthritis. Arthritis. 2011; 2011:765624. doi: 10.1155/2011/765624. Epub 2011 May 26.

[22] Dayer JM, Choy E. Therapeutic targets in rheumatoid arthritis: the interleukin-6 receptor. Rheumatology 2010; 49:15- 24.

[23] Smolen JS, Aletaha D, Koeller M, Weisman MH, Emery P. New therapies for treatment of rheumatoid arthritis. Lancet 2007; 370:1861- 74 . 